\title{
Expanding the Phenotypic and Genetic Spectrum of Neuromuscular Diseases Caused by DYNC1H1 Mutations
}

\author{
Jia-Tong Li $^{1}$, Si-qi Dong ${ }^{1}$, and Xiangjun Chen ${ }^{1}$ \\ ${ }^{1}$ Huashan Hospital Fudan University
}

June 4, 2021

\begin{abstract}
Objective: Spinal muscular atrophy, lower limb-dominant (SMA-LED), and Charcot-Marie-Tooth type 2O (CMT2O) are two kinds of familial neuromuscular diseases. In this study, we reported two patients with CMT2O caused by DYNC1H1 mutations and further analyzed the genotype-phenotype correlations. Methods: Two CMT2O patients and their parents' clinical data were collected and genetic analysis was applied. PubMed, Web of Science, CNKI and Wanfang Data were searched, and all publications that met the inclusion criteria were carefully screened. Results: Genetic testing of our two CMT2O patients revealed heterozygous mutations DYNC1H1 c.1792C>T/p.R598C and c.790C > G/p.R264G, respectively. Next, a total of 22 original published articles were included and analyzed. Compared to SMA-LED, CMT2O patients had no proximal-dominant wasting, more distal-dominant weakness of lower limbs and more sensory abnormalities. Genotype-phenotype analysis revealed that mutations in the DYN1 region of DYNC1H1 protein were associated with a more severe phenotype, more complicated symptoms and more involvement of the central nervous system than that in the DHC_N1 region. Conclusion: Our findings of the phenotypic differences between SMA-LED and CMT2O patients provide references for early diagnosis and differentiation of the two diseases. The genotype-phenotype correlation may reflect the pathogenesis underlying dyneinopathy caused by DYNC1H1 mutations.
\end{abstract}

Expanding the Phenotypic and Genetic Spectrum of Neuromuscular Diseases Caused by DYNC1H1 Mutations

Jia-Tong Li ${ }^{1}$, Si-Qi Dong ${ }^{1}$, Xiang-Jun Chen ${ }^{1,2}$ (ORCID:0000-0002-8108-9013)

Department of Neurology, Institute of Neurology, Huashan Hospital Fudan University, Shanghai 200040, P.R. China

Human Phenome Institute, Fudan University, Shanghai 200433, P.R. China

Corresponding author: Xiang-Jun Chen, Department of Neurology, Huashan Hospital, Fudan University, 12 Wulumuqi Zhong Road, Shanghai 200040, China, Tel: +86-21-52888159, Fax: 86-21-62491694, E-mail:

xiangjchen@fudan.edu.cn

Grant numbers: No.SKLCB2018KF004, 2018SHZDZX01

\begin{abstract}
Objective: Spinal muscular atrophy, lower limb-dominant (SMA-LED), and Charcot-Marie-Tooth type $2 \mathrm{O}$ (CMT2O) are two kinds of familial neuromuscular diseases. In this study, we reported two patients with CMT2O caused by DYNC1H1 mutations and further analyzed the genotype-phenotype correlations.
\end{abstract}


Methods: Two CMT2O patients and their parents' clinical data were collected and genetic analysis was applied. PubMed, Web of Science, CNKI and Wanfang Data were searched, and all publications that met the inclusion criteria were carefully screened.

Results: Genetic testing of our two CMT2O patients revealed heterozygous mutations DYNC1H1 c.1792C > T/p.R598C and c.790C >G/p.R264G, respectively. Next, a total of 22 original published articles were included and analyzed. Compared to SMA-LED, CMT2O patients had no proximal-dominant wasting, more distal-dominant weakness of lower limbs and more sensory abnormalities. Genotype-phenotype analysis revealed that mutations in the DYN1 region of DYNC1H1 protein were associated with a more severe phenotype, more complicated symptoms and more involvement of the central nervous system than that in the DHC_N1 region.

Conclusion: Our findings of the phenotypic differences between SMA-LED and CMT2O patients provide references for early diagnosis and differentiation of the two diseases. The genotype-phenotype correlation may reflect the pathogenesis underlying dyneinopathy caused by DYNC1H1 mutations.

Keywords Neuromuscular diseases, Spinal muscular diseases, Charcot-Marie-Tooth disease, DYNC1H1, Genotype-phenotype correlation

\section{Introduction}

Dynein cytoplasmic 1 heavy chain 1 (DYNC1H1), located on 14q32.31, is the core structure of cytoplasmic dynein, which is a large ( $\sim 1.5 \mathrm{MDa})$ motor protein complex responsible for retrograde axonal transport in all eukaryotic cells (Pfister et al., 2006). DYNC1H1 plays an essential role in ATPase-dependent movement along the microtubule and recruitment of other dynein subunits (Pfister et al., 2006). Mutations in DYNC1H1 can lead to various developmental and degenerative diseases of the nervous system, which are referred to as "dyneinopathy" characterized by locomotor and motor system deficits, sensory system defects, and (or) brain morphology and function abnormalities (Marzo et al., 2019).

Spinal muscular atrophy lower limb-dominant (SMA-LED, OMIM \#158600) and Charcot-Marie-Tooth type $2 \mathrm{O}$ (CMT2O, OMIM \#614228) have both been reported as hereditary neuromuscular diseases caused by DYNC1H1 variants (Harms et al., 2012); (Tsurusaki et al., 2012); (Weedon et al., 2011). Spinal muscular atrophy (SMA) is caused by the impairment of motor neurons in the spinal cord. SMA-LED is an autosomal dominant hereditary type of SMA characterized by muscle weakness and wasting predominantly affecting the lower limbs (Harms et al., 2010). Charcot-Marie-Tooth (CMT) disease, also known as hereditary motor and sensory neuropathies (HMSN), is a genetically heterogeneous disease, clinically characterized by progressive distal muscle weakness and wasting, sometimes accompanied by sensory abnormalities. CMT2O was firstly reported by Weedon et al. in a large four-generation family and the patients presented with delayed motor milestones, abnormal gait and slowly progressive distal lower limb atrophy and weakness accompanied with pes cavus deformity (Weedon et al., 2011), as we proposed the human mutation counterpart through mapping the $S w l$ allele in a dync1h1 mutation animal model (Chen et al., 2007).

SMA-LED and CMT2O share many common clinical phenotypes and will sometimes be misdiagnosed. Although numerous cases have been reported worldwide, the analysis on their manifestation difference and genotype-phenotype correlation is still limited. In this study, we first reported DYNC1H1 gene c.1792C $>$ T/p.R598C and c.790C $>$ G/p.R264G pathogenic mutations in two CMT $2 \mathrm{O}$ cases, respectively. Next, we reviewed previous publications and described the phenotypic and genetic characteristics of neuromuscular diseases caused by DYNC1H1 mutations and compared the clinical phenotypes between SMA-LED and CMT2O. Furthermore, we focused on the genotype-phenotype correlation of DYNC1H1 variants to provide references for making personalized clinical decisions in clinical practice.

\section{Methods}

Ethics

The study was approved by the ethics committee of Huashan Hospital, Fudan University. The patients and 
their family members all went through the informed consent process.

Genetic analysis

Peripheral blood was collected and sent to the whole-exome sequencing (Illumina HiSeq 4000, Illumina, San Diego, CA). The exons of CMT 2O-related genes were detected using the second-generation highthroughput sequencing technology. To find the gene variation, the sequencing data and the human reference GRCh37/hg19 were sequenced and analyzed. These gene variations were annotated based on the standardized bioinformatics algorithm flow, including Clinvar, dbSNP database, and gnomAD database.

Analysis and interpretation of clinical data

Sanger sequencing was carried out for gene variations that may correlate with the clinical phenotype of subjects. The pathogenicity of gene variations is classified into five degrees: "pathogenic", "possibly pathogenic", "uncertain significance", "possibly benign", and "benign" according to the classification standard of the American College of Medical Genomics (ACMG) (Richards et al., 2015).

Collection of previous publications

PubMed, Web of Science, CNKI and Wanfang Data were searched, applying the following search terms from January 1980 to April 2021: (mutation OR variant) AND (DYNC1H1 AND ("Charcot-Marie-Tooth Disease" OR "CMT" OR "hereditary motor and sensory neuropathy" OR "HMSN" OR "spinal muscular atrophy" OR "SMA")). Human Gene Mutation Database (HGMD) (http://www.hgmd.cf.ac.uk/), Online Mendelian Inheritance in Man (OMIM) (https://www.omim.org/) and Ensembl (http://uswest.ensembl.org/index.html) were also searched with the search term DYNC1H1 . All resulting publications were carefully screened. Publications that met the following criteria were included: (1) were studies involving patients with neuromuscular diseases, typically SMA-LED and CMT2O, (2) provided the exact mutation in $D Y N C 1 H 1$, and (3) full-text available. We also excluded publications that: (1) were reviews, (2) were not case reports, (3) did not involve neuromuscular diseases and (4) did not focus onDYNC1H1 variants. Among included publications, families or a single patient that had no detailed description of clinical phenotypes were also excluded from this study to improve the quality for analysis. Clinical and genetic data were collected from each reported patient, including age, gender, origin, clinical manifestations, disease onset age, mutation location and variant type, etc. All the variants were checked or adjusted to ensure that they matched with reference transcript NM_001376.5 for DYNC1H1 . All the processes were performed independently by two authors and any discrepancy in the assessment would be resolved by consensus.

\section{Statistics}

The distributions of continuous variables were tested for normality with the Kolmogorov-Smirnov test, and the difference between two groups was compared by student's t-test or Mann-Whitney test wherever appropriate. Chi-squared test or Fisher exact test was used to compare the categorical variables. Moreover, Bonferroni adjustment was also applied in multiple comparisons. All analyses were 2 -tailed, and $P$ value $<$ 0.05 was considered statistically significant. All the statistical data were analyzed using SPSS 20.0 (SPSS Inc., Chicago, IL, USA).

\section{Results}

\section{Clinical phenotypic and genetic analysis of two CMT2O cases}

Case 1 is a 14-year-old female patient who complained of difficulty standing upright from a squatting position for over one year and came to Huashan Hospital, Fudan University for further examination. Her mother declared that the patient learned to walk at 14 months of age and ran slower than her peers since childhood. On physical examination, the patient presented with pes cavus and muscle wasting of distal lower limbs. Muscle strength was normal except for $4 / 5$ weaknesses of distal upper limbs and $4 / 5$ bilateral lower limbs weaknesses. Tendon reflexes were attenuated in all limbs, and Babinski's sign was negative. The sensory system was normal on examination. The patient also showed an unaffected intelligence level. Her parents presented normal, and there was no related family history (Fig.1A). 
Case 2 is a 15-year-old male patient who came to Huashan Hospital, Fudan University, due to the noticeable thinning of lower limbs for two years. His father complained that the patient could walk at 19 months of age and had been prone to fall since childhood. Physical examination showed mild lordosis and muscle wasting of both lower limbs. Muscle strength was normal except for $4 / 5$ weakness of bilateral distal lower limbs. Tendon reflexes were attenuated. Babinski's sign was absent. The patient's sensory examination was normal with an unaffected intelligent level. His parents were both normal, and no related family history was declared (Fig.1B).

Laboratory examinations revealed normal (181 U/L) and elevated (294 U/L) level of serum creatine kinase in the two patients, respectively. In case 1, electromyogram (EMG) suggested anterior spinal horn deterioration, and the involvement of motor nerve axons could not be excluded as well. And in case 2, EMG showed chronic neurogenic damage with consideration of impairment of anterior horn motor neurons or anterior spinal root. The nerve conduction velocity (NCV) test, motor nerve $\mathrm{F}$ wave latency, $\mathrm{H}$ reflex latency and repetitive nerve stimulation (RNS) revealed normal results in both patients. In the second patient, MR scan of the femur showed a partial fat signal suggesting muscle atrophy of the right thigh muscle group and a small amount of effusion in the right hip joint cavity. Biopsy of the left sural nerve revealed that the pathological changes were consistent with pathological characteristics of chronic axonal peripheral neuropathy.

Whole-exome sequencing discovered DYNC1H1 c.1792C > T/p.R598C and DYNC1H1 c.790C $>$ G/p.R264G heterozygous mutations in the two patients, respectively, the latter of which is a novel mutation site that has not been reported before. Sanger sequencing revealed that the parents of both patients did not carry these point mutations (Fig.1A, B), indicating these mutations de novo. Bioinformatics software (Polyphen2, SIFT, Mutation Taster) and ACMG guidelines predicted both mutations as "pathogenic" (Fig.1C).

\section{Spectrum of $D Y N C 1 H 1$ mutations in neuromuscular diseases}

Previous publications were searched to further investigate the clinical and genetic spectrum of neuromuscular diseases caused by DYNC1H1 mutations. A total of 22 original articles out of 49 publications met the inclusion criteria and were included in our study (Fig.S1). These articles identified 39 variants in DYNC1H1 related to neuromuscular diseases. Of the 105 patients, SMA-LED (77, 73.3\%) and CMT2O (16, 15.2\%) accounted for the most part. Among the SMA-LED patients, two were initially diagnosed with polio and chronic inflammatory demyelinating polyradiculoneuropathy (CIDP), respectively. Other types of neuromuscular diseases, such as hereditary spastic paraplegia (HSP), were also reported caused by DYNC1H1 variants. All variants were missense mutations, except for one splicing mutation (c.12685-3C $>\mathrm{T}$ ). One hundred five patients from 58 pedigrees were included, among which all carried one heterozygous variant in $D Y N C 1 H 1$, except for two patients from a Chinese family harbouring two variants in $D Y N C 1 H 1$, including c.2419G $>$ A/p.G807S and c.12685-3C $>$ T. Of all the 58 families from all over the world, most were European families $(34 / 58,58.6 \%)$, followed by East Asian (9/58, 15.5\%), North American (8/58, 13.8\%), Australian $(5 / 58,8.6 \%)$ and Middle East $(2 / 58,3.4 \%)$.

\section{Characteristics of $D Y N C 1 H 1$ mutation spectrum}

Of all the DYNC1H1 variants, exon 8 was found to be the mutational hot region with $24 / 58(41.4 \%)$ families including 52 patients. Other clustered variants distributed in exon 4 ( $7 / 58$ families with 7 patients, 12.1\%), exon 5 (5/58 families with 19 patients, $8.6 \%$ ), exon 6 (2/58 families with 3 patients, $3.4 \%)$, exon $13(2 / 58$ families with 3 patients, $3.4 \%$ ), exon 14 (3/58 families with 3 patients, $5.2 \%$ ), and exon 38 (2/58 families with 3 patients, 3.4\%) (Fig.2). Several recurrent DYNC1H1 variants were reported, with c. $1792 \mathrm{C}>\mathrm{T} / \mathrm{p} . \mathrm{R} 598 \mathrm{C}$ being the most common mutation $(7 / 58$ families with 10 patients, $12.1 \%)$. Others were c.751C $>\mathrm{T} / \mathrm{p} . \mathrm{R} 251 \mathrm{C}$ (6/58, 10.3\%), c.1953G>A/p.V612M (4/58 6.9\%), c.2327C>T/p.P776L $(3 / 58,5.2 \%)$, c.917A $>$ G/p.H306R $(3 / 58,5.2 \%)$ and c.3170A $>$ G/p.Y970C $(2 / 58,3.4 \%)$. Interestingly, although c.1792C $>$ T/p.R598C remains the most common mutation site related to neuromuscular diseases worldwide, it has not been reported in East Asian population yet. Instead, c.751C > T/p.R251C and c.2327C $>$ T/p.P776L were more commonly reported in this region, which were found in $3 / 9(33.3 \%)$ families and 2/9 $(22.2 \%)$ families respectively.

Human DYNC1H1 protein is composed of 4646 amino acids (aa). Traditionally, it can be divided into the 
tail domain (0-1450 aa) and the motor domain (1450-4646 aa) according to its structural and functional characteristics. Of all the $39 \mathrm{DYNC1H1}$ variants, $27 / 39(69.2 \%)$ were distributed in the tail domain and only $12 / 39(30.8 \%)$ located in the motor domain, which was in correspondence with a previous study that mutations in the tail domain were more commonly related to motor-related disorders (Harms et al., 2012); (Rossor, Kalmar, Greensmith, \& Reilly, 2012). More exactly, the DYNC1H1 protein is divided into several domains overlapping each other (Fig.3). Cases involved in our study revealed that the stem domain (53-1867 aa) contained the most amount of DYNC1H1 mutations (30/39, 76.9\%), among which 18/39 (46.2\%) were located in overlapping regions between stem and other domains, including DHC_N1 (242-832 aa), dynein intermediate chain (DIC)-binding domain (448-703 aa) and dynein intermediate light chain (DILC-binding) domain (651-802 aa). Mutations in other domains were relatively less frequent, including AAA2 (2180-2452 aa, 1/39, 2.6\%), AAA3 (2556-2805 aa, 3/39, 7.7\%), AAA4 (2899-3168 aa, 2/39, 5.1\%), stalk (3189-3500 aa, $2 / 39,5.1 \%)$ and the region between AAA4 and stalk $(1 / 39,2.6 \%)$. This result may be due to the fact that stem is the longest domain of DYNC1H1 located more closely to the N-terminal and overlaps many other regions. The detailed information of $D Y N C 1 H 1$ mutation spectrum of patients with neuromuscular diseases was listed in table S1.

\section{Clinical phenotypes of neuromuscular diseases caused by DYNC1H1 mutation}

A total of 105 patients with neuromuscular diseases were reported carrying DYNC1H1 mutations, among which 103 (43 males, 27 females and 33 genders not mentioned) harbored one heterozygous variant in DYNC1H1 . In comparison, the other two patients (a young male and his mother) had two variants. Of 66 patients with exact ages provided, the average age was 21.8 19.7 years old (range, $1.0-82.0$; median, 15.5). The onset age of the patients with DYNC1H1 mutations was relatively young, with 34/90 $(37.8 \%)$ at birth, $27 / 90(30.0 \%)$ in infancy ( $<1$ year), 14/90 (15.6\%) in early childhood (1-3 years), 7/90 $(7.8 \%)$ in childhood (3-12 years), 1/90 (1.1\%) at juvenile (12-18 years) and 7/90 (7.8\%) in adulthood (>18 years) (Fig.4A). Muscle weakness of lower limbs was the most common clinical phenotype of these patients (98/102 patients, 96.1\%). The distribution of lower limb weakness was different among patients, with $44 / 68(64.7 \%)$ presented with proximal-dominant weakness, 16/68 (23.5\%) had distal-dominant weakness, and 10/68 (14.7\%) showed similar severity of muscle weakness between proximal and distal lower limbs. Although the incidence of weakness was high, most patients remained ambulant (89/102, 87.3\%), and Gower's sign was not common in patients $(9 / 54,16.7 \%)$, suggesting the relatively mild degree of lower limb and trunk muscle impairment. Muscle wasting of lower limbs was also a predominant clinical phenotype with 83/98 (84.7\%) patients involved, among which more patients $(34 / 55,61.8 \%)$ showed distal-dominant atrophy compared to proximal-dominant atrophy $(11 / 55,20.0 \%)$. A small part of patients $(18 / 97,18.6 \%)$ also had upper limb involvement. However, the involvement of trunk muscles was rare, which was observed in 5/97 $(5.2 \%)$ patients. Delayed motor milestones were usually the earliest clinical manifestation of patients with SMA-LED or CMT2O and were reported in 54/90 (60\%) patients. The sensory system was usually exempted from the diseases, with 10/84 (11.9\%) presented with sensory abnormalities. Most patients (91/101, 90.1\%) showed deformities that predominantly involving the musculoskeletal system. Of these abnormalities, joint contracture or deformities occurred in 36/91 (39.6\%) patients. Pes cavus occurred in 37/91 (40.7\%) patients and pes planus was observed in 6/91 (6.6\%) patients. In addition, 26/91 (28.6\%) patients had other kinds of feet deformities. There were 3/91 (3.3\%) patients and 25/91 (27.5\%) patients presenting with hand and spine deformities, respectively. Other kinds of abnormalities involving other body parts were also found in 20/91 (22.0\%) patients, including scapular winging, high-arch palate, exotropia, retinoblastoma, strabismus, bilateral congenital cataract, aortic root dilation, accessory spleen and facial dysmorphia, etc. The involvement of the central nervous system (CNS) was relatively less common. Seizure episode occurred in 11/101 $(10.9 \%)$ patients. About a third patients $(31 / 94,33.0 \%)$ had abnormal intelligence or delayed intelligence development and 13/94 (13.8\%) were diagnosed as attention deficit hyperactivity disorder (ADHD). The impairment of intelligence was mostly mild $(17 / 21,81.0 \%)$, followed by severe $(3 / 21,14.3 \%)$ and moderate $(1 / 21,4.8 \%)$ level. The percentage of some clinical phenotypes were shown in Fig.4B.

The most notable clinical phenotype on physical examination was abnormal tendon reflex in lower limbs, which was observed in 75/90 (83.3\%) patients. Most of these patients $(73 / 75,97.3 \%)$ showed decreased or 
absent tendon reflex, while increased tendon reflex was also found $(2 / 75,2.7 \%)$. Serum creatine kinase (CK) level only increased in $3 / 24(12.5 \%)$ patients. The nerve conduction velocity (NCV) test usually showed normal results, with only 10/57 (17.5\%) exceptions. In addition, abnormal brain MRI was detected in 18/30 $(60.0 \%)$ patients. The detailed information of clinical phenotypes of patients with neuromuscular diseases caused by $D Y N C 1 H 1$ mutations was listed in table S2.

\section{Comparison of clinical phenotypes between SMA-LED and CMT2O}

Clinical phenotypes of SMA-LED and CMT2O were compared in Table 1. The two groups of patients showed different onset age $(P=0.006)$, with more SMA-LED patients $(30 / 63,47.6 \%)$ were born with clinical manifestations, while more CMT2O patients $(7 / 15,46.7 \%)$ had the disease onset in infancy ( $<1$ year). Besides, no CMT2O patient had their disease onset in adulthood compared to $7 / 63$ (11.1\%) SMA-LED patients. Seven out of $73(9.6 \%)$ cases of epilepsy were observed in SMA-LED patients but none was found in $\mathrm{CMT} 2 \mathrm{O}$ patients, although the result was of no statistical significance $(P=0.437)$. The distribution pattern of muscle atrophy differed between SMA-LED and CMT2O patients. Although both groups of patients more commonly presented distal-dominant lower limb atrophy, none of the CMT2O patients had proximal-dominant wasting compared to $11 / 42(26.2 \%)$ SMA-LED patients $(P<0.05)$. In addition, the distribution of muscle weakness in lower limbs showed a more significant difference between the two groups $(P<0.001)$. Interestingly, 39/48 (81.3\%) SMA-LED and 1/16 (6.2\%) CMT2O patients showed proximaldominant lower limb weakness $(P<0.05)$, respectively, while more CMT2O patients $(13 / 16,81.3 \%)$ had distal-dominant manifestation compared to SMA-LED $(3 / 48,6.2 \% ; P<0.05)$. Abnormal sensation was more common in CMT2O than SMA-LED patients $(9 / 16,56.3 \%$ and $1 / 66,1.5 \%$ respectively, $P<0.001)$. As for CNS involvement, both SMA-LED and CMT2O patients had a similar incidence of cognitive impairment $(17 / 66,25.8 \%$ and $4 / 16,25.0 \%$, respectively). ADHD was observed in 9/66 (13.6\%) SMA-LED patients compared to none of the CMT2O patient, although there was no statistical significance $(P=0.263)$. Besides, seven out of $34(20.6 \%)$ SMA-LED patients showed abnormal NCV results, while all CMT2O patients were normal on $\operatorname{NCV}(P=0.128)$.

\section{Genotype-phenotype correlations of $D Y N C 1 H 1$ variants}

Generally, the DYNC1H1 protein can be divided into the N-terminal region named DHC_N1 (242-832 aa), the C-terminal region named Dynein_C (4333-4633 aa) and the longest DYN1 region (1046-4329 aa) in between. Of all the DYNC1H1 mutations leading to neuromuscular diseases, none was located in Dynein_C. Therefore, we divided these mutations into two groups according to their locations: the DHC_N1 group (p.G192R also included) and the DYN1 group (p.Y970C also included). The comparison of the phenotypes correlated with these two genotypes was listed in Table 2. Atrophy and weakness of lower limbs were both common in the two groups, and both groups had more cases of distal-dominant atrophy and proximal-dominant weakness. However, the severity of these symptoms between the two groups showed some differences, with more severe lower limb symptoms lying in the DYN1 group $(5 / 18,27.8 \%$ v.s. $3 / 5,60.0 \% ; P>0.05$ and $4 / 22,18.2 \%$ v.s. $7 / 12,58.3 \% ; P<0.05)$. Delayed motor milestones were more commonly observed in the DYN1 group than the DHC_N1 group $(18 / 23,78.3 \%$ v.s. $36 / 67,53.7 \%, P=0.038)$. Besides, the DYN1 group also had a higher percentage of upper limb involvement, trunk involvement, deformities and abnormal tendon reflex, as well as a lower percentage of ambulant patients, although the results were not statistically significant. Abnormal NCV results were also significantly more common in the DYN1 group than in the DHC_N1 group (6/12, $50 \%$ v.s. $4 / 45,8.9 \%, P=0.004)$. These results indicated that mutations in the DYN1 region might present a more severe phenotype and more diverse clinical manifestations than the DHC_N1 mutations.

Mutations in the DYN1 region was more related to CNS impairment as well. More epilepsy was observed in the DYN1 group $(6 / 24,25.0 \%)$ than in the DHC_N1 group $(5 / 77,6.5 \% ; P=0.030)$. The incidence of cognitive impairment was significantly higher in the DYN1 group $(15 / 19,78.9 \%$ v.s. $16 / 75,21.3 \%, P<0.001)$ and ADHD was more common in this group, although the difference was not significant $(5 / 19,26.3 \%$ v.s. $8 / 75$, $10.7 \% ; P=0.164)$. In accordance with this, abnormalities of the brain were more commonly found on MRI of the DYN1 group $(12 / 14,85.7 \%)$ compared to the DHC_N1 group $(6 / 17,35.3 \% ; P=0.005)$. 


\section{Discussion}

SMA-LED and CMT2O are related to DYNC1H1 mutations and are characterized by predominant wasting and weakness of lower limbs, delayed motor milestones, and abnormalities on electromyography (EMG) examination. In this study, we reported two CMT2O cases with heterozygous DYNC1H1 mutations c. $1792 \mathrm{C}>\mathrm{T} / \mathrm{p} . \mathrm{R} 598 \mathrm{C}$ and c.790C $>$ G/p.R264G, respectively. The former mutation of DYNC1H1 is, to our knowledge, the first one reported in East Asia that causes CMT 2O, and the latter is a novel dominant mutation reported for the first time worldwide. In addition, we analyzed the clinical phenotypes of neuromuscular diseases caused by $D Y N C 1 H 1$ mutations and relevant genotype-phenotype correlation in previous publications. We compared the clinical characteristics between the two disease entities. We found that all CMT2O patients were presented with distal-dominant wasting, while proximal-dominant lower limb wasting can be observed in some cases of SMA-LEDs. We also found that patients with mutations in the DYN1 region may have more severe phenotype than those mutated in the DHC_N1 region.

Our results showed that weakness and wasting of lower limbs were the most common clinical phenotype of DYNC1H1- related neuromuscular diseases. Delayed motor milestones were also observed in a large percentage of patients. The most common phenotype on physical examination was decreased or absent tendon reflex. This was in accordance with previous animal experiments that the Swlheterozygous mutation of Dync1h1 led to absent $\mathrm{H}$ reflex in mice, even if the motor nerve function was normal (Chen et al., 2007).

The most common clinical features of SMA-LED and CMT2O, including delayed motor milestones and lower limb-predominant wasting and weakness, are often not specific, especially in the early stage of the diseases. Typically, SMA-LED and CMT2O are diagnosed and differentiated according to EMG results. On EMG examination, SMA-LED is characterized by motor axonal or motor neuron loss, which is in the form of active denervation, compensatory reinnervation and enlargement of the motor unit action potential (Arnold, Kassar, \& Kissel, 2015). CMT2O usually presents a typical pattern of axonal neuropathy affecting both motor and sensory modalities on EMG, similar to other CMT type2 diseases (Pakhrin et al., 2018). Our study also suggested the NCV test as another applicable electrophysiological approach to differentiate these disease entities, as abnormal NCV result may be more commonly seen in SMA-LED than that in CMT2O. However, in the early stage of these diseases, electrophysiological abnormalities may not be typical enough to make an accurate diagnosis. Therefore, considering the common variants of gene that may lead to SMA-LED and CMT2O, clinical phenotypes may be an essential characterization in the early diagnosis and differentiation of these two disease entities. In our study, it was found that all CMT2O patients were presented with distaldominant wasting, while proximal-dominant lower limb wasting can be observed in some cases of SMA-LEDs. Besides, SMA-LED patients had a higher percentage of proximal-dominant weakness of lower limbs, while CMT2O patients more frequently presented distal-dominant weakness. This may suggest that although lower limb involvement is a common feature of both diseases, they may have different distribution pattern of muscle impairment, which may provide some insights for clinicians when making an early diagnosis. In addition, abnormal sensation was more common in CMT2O patients, consistent with earlier reports that SMA-LED patients are usually exempt from sensory impairment (Arnold et al., 2015). Therefore, the involvement of the sensory system may be a relatively strong indicator of $\mathrm{CMT} 2 \mathrm{O}$ in patients with $D Y N C 1 H 1$ mutation presenting relevant clinical manifestations.

DYNC1H1 mutations involved in our study were located either in the DHC_N1 region or in the DYN1 region. Not only did patients with mutations in DYN1 present with more severe atrophy and weakness of lower limbs, but they may also show clinical phenotypes more complicated and difficult to make a right diagnosis. Besides, manifestations of CNS impairment, such as epilepsy, cognitive impairment, ADHD and abnormal brain MRI was more common in patients with mutations in the DYN1 region. This is in accordance with earlier finding that mutations in the tail domain (close to the N-terminal) usually lead to pure motor neuron deficits hardly with any signs of brain abnormalities (Harms et al., 2012); (Rossor et al., 2012), while mutations in the motor domain (close to the C-terminal) often cause malformation of cortical development (MCD) and epilepsy (Poirier et al., 2013). Interestingly, no DYNC1H1 mutation related to motor neuron diseases has been observed located in the C-terminal (Dynein_C region). This suggests a highly clinical heterogeneity of 
$D Y N C 1 H 1$ variants, the mechanism of which warrants further investigation, such as epigenetic modification, environmental exposure, etc. In addition, as precision medicine is becoming more and more advocated, this genotype-phenotype correlation of $D Y N C 1 H 1$ mutation may serve as a reference for accurate diagnosis and personalized treatment.

Our study also suggested that some mutation sites of DYNC1H1 had a higher recurrence rate than other sites, of which exon 8 seemed to be the mutational hot region and c.1792C $>$ T/p.R598C was the most frequent point mutation. However, no c.1792C $>$ T/p.R598C mutation has been found in the East Asian population at present, although some other recurrent DYNC1H1 mutations have been detected in East Asia. On one hand, this discrepancy may reflect differences on genetic level among different races; on the other hand, this result may be due to the relatively limited amount of cases in this area. Considering the large population basis in East Asia, more different kinds of DYNC1H1 mutations may be gradually detected, as our team recently identified a teenage patient with CMT2O caused by c. $1792 \mathrm{C}>\mathrm{T} / \mathrm{p} . \mathrm{R} 598 \mathrm{C}$ heterozygous mutation in DYNC1H1 . Up to now, no specific treatment for SMA-LED and CMT2O has been put forward yet, thus symptomatic treatment and orthopedic surgery remain important in order to decrease the risk of complications of these diseases as early as possible. Therefore, genetic testing is of necessity in early diagnosis and prompt treatment. Besides, gene treatment, such as genome editing, provides new insight into treating diseases caused by pathogenic gene mutations, especially those with high recurrent rate. In this regard, early genetic testing for clinically suspected SMA-LED or CMT2O patients will be illustrating for applying appropriate therapeutics and trying new therapeutic techniques.

The conclusions of this study shouldn't be assessed without the consideration of several limitations. Firstly, not all patients have a well-recorded phenotype, though the literature were systematically reviewed. Thus, the study did not present a complete mutation spectrum since patients without any detailed description of clinical features are not studied. Secondly, the reporting bias of included articles cannot be ignored because cases with recurrent variants and classical phenotypes were less likely to be reported. Thirdly, all these comparisons are based on cases in the literature and online database. Therefore, the genotype-phenotype correlations should be validated in an independent cohort. Despite the limitations above, our study provided a comprehensive summary of the DYNC1H1 mutations spectrum by selecting SMA-LED and CMT2O diseases and unrevealed the hidden correlation between genotype and phenotype.

\section{Conclusions}

In this study, we reported two CMT $2 \mathrm{O}$ cases carrying DYNC1H1 c.1792C $>\mathrm{T} / \mathrm{p} . \mathrm{R} 598 \mathrm{C}$ and a novel site, DYNC1H1 c.790C $>$ G/p.R264G heterozygous mutations, respectively, clinically manifesting delayed motor milestones, muscle weakness and wasting of lower limbs and normal sensation. By systematically reviewing previous publications, we found that SMA-LED and CMT2O patients caused by DYNC1H1 mutations showed generally similar clinical phenotypes with some differences that may provide references for early diagnosis and differentiation of the two disease entities, such as the differences in the distribution pattern of muscle impairment, sensory system involvement, etc. Our findings also suggested that mutations in the DYN1 region of DYNC1H1 may be correlated with more severe clinical phenotype and more complicated manifestations. In addition, CNS involvement is also more common in such patients. The genotype-phenotype correlation may reflect the pathogenesis underlying dyneinopathy caused by DYNC1H1 mutations.

\section{Acknowledgments}

The authors acknowledge the patients and their families for their cooperation. The authors thank Prof. Dong-sheng Fan and Ze-xu Chen for their kind assistance in critically revising the manuscript.

Author contributions

All authors designed the study and collected the clinical and genetic data of the two patients. Jia-tong Li and Si-qi Dong completed the collection and assessment of previous publications independently and wrote the manuscript. Xiang-jun Chen revised the manuscript. All authors read and approved the manuscript.

\section{Funding}


Funding for this study is provided by the Open Project of State Key Laboratory of Cell Biology (No.SKLCB2018KF004),2020 Central Transfer Payment Medical Siege Institutions Capacity Building Project (National and Provincial Multi-scientific Cooperation Diagnosis and Treatment of Major Diseases Capacity Building Project) and the Shanghai Municipal Science and Technology Major Project [2018SHZDZX01] and ZJLab.

\section{Conflict of Interest Statement}

The authors declare no conflicts of interest. The authors alone are responsible for the content and writing of this article.

\section{Ethics}

The study was approved by the ethics committee of Huashan Hospital, Fudan University. The patients and their family members all went through the informed consent process.

\section{Data availability statement}

All data relevant to the study are included in the article or uploaded as supplementary information.

\section{References:}

Arnold, W. D., Kassar, D., \& Kissel, J. T. (2015). Spinal muscular atrophy: diagnosis and management in a new therapeutic era. Muscle Nerve, 51, 157-167. doi: 10.1002/mus.24497

Chen, X. J., Levedakou, E. N., Millen, K. J., Wollmann, R. L., Soliven, B., \& Popko, B. (2007). Proprioceptive sensory neuropathy in mice with a mutation in the cytoplasmic Dynein heavy chain 1 gene. J Neurosci , 27, 14515-14524. doi: 10.1523/JNEUROSCI.4338-07.2007

Harms, M. B., Allred, P., Gardner, R. J., Fernandes, F. J., Florence, J., Pestronk, A., Al-Lozi, M., \& Baloh, R. H. (2010). Dominant spinal muscular atrophy with lower extremity predominance: linkage to 14q32.Neurology , 75, 539-546. doi: 10.1212/WNL.0b013e3181ec800c

Harms, M. B., Ori-McKenney, K. M., Scoto, M., Tuck, E. P., Bell, S., Ma, D., Masi, S., Allred, P., Al-Lozi, M., Reilly, M. M., Miller, L. J., Jani-Acsadi, A., Pestronk, A., Shy, M. E., Muntoni, F., Vallee, R. B., \& Baloh, R. H. (2012). Mutations in the tail domain of DYNC1H1 cause dominant spinal muscular atrophy. Neurology , 78, 1714-1720. doi: 10.1212/WNL.0b013e3182556c05

Marzo, M. G., Griswold, J. M., Ruff, K. M., Buchmeier, R. E., Fees, C. P., \& Markus, S. M. (2019). Molecular basis for dyneinopathies reveals insight into dynein regulation and dysfunction. Elife, 8. doi: 10.7554/eLife.47246

Pakhrin, P. S., Xie, Y., Hu, Z., Li, X., Liu, L., Huang, S., Wang, B., Yang, Z., Zhang, J., Liu, X., Xia, K., Tang, B., \& Zhang, R. (2018). Genotype-phenotype correlation and frequency of distribution in a cohort of Chinese Charcot-Marie-Tooth patients associated with GDAP1 mutations.J Neurol , 265, 637-646. doi: 10.1007/s00415-018-8743-9

Pfister, K. K., Shah, P. R., Hummerich, H., Russ, A., Cotton, J., Annuar, A. A., King, S. M., \& Fisher, E. M. (2006). Genetic analysis of the cytoplasmic dynein subunit families. PLoS Genet, 2, e1. doi: 10.1371/journal.pgen.0020001

Poirier, K., Lebrun, N., Broix, L., Tian, G., Saillour, Y., Boscheron, C., Parrini, E., Valence, S., Pierre, B. S., Oger, M., Lacombe, D., Genevieve, D., Fontana, E., Darra, F., Cances, C., Barth, M., Bonneau, D., Bernadina, B. D., N'Guyen, S.,... Chelly, J. (2013). Mutations in TUBG1, DYNC1H1, KIF5C and KIF2A cause malformations of cortical development and microcephaly. Nat Genet , 45, 639-647. doi: $10.1038 /$ ng. 2613 
Richards, S., Aziz, N., Bale, S., Bick, D., Das, S., Gastier-Foster, J., Grody, W. W., Hegde, M., Lyon, E., Spector, E., Voelkerding, K., \& Rehm, H. L. (2015). Standards and guidelines for the interpretation of sequence variants: a joint consensus recommendation of the American College of Medical Genetics and Genomics and the Association for Molecular Pathology. Genetics in Medicine , 17, 405-423. doi: $10.1038 / \operatorname{gim} .2015 .30$

Rossor, A. M., Kalmar, B., Greensmith, L., \& Reilly, M. M. (2012). The distal hereditary motor neuropathies. J Neurol Neurosurg Psychiatry , 83, 6-14. doi: 10.1136/jnnp-2011-300952

Schiavo, G., Greensmith, L., Hafezparast, M., \& Fisher, E. M. (2013). Cytoplasmic dynein heavy chain: the servant of many masters. Trends Neurosci , 36, 641-651. doi: 10.1016/j.tins.2013.08.001

Tsurusaki, Y., Saitoh, S., Tomizawa, K., Sudo, A., Asahina, N., Shiraishi, H., Ito, J., Tanaka, H., Doi, H., Saitsu, H., Miyake, N., \& Matsumoto, N. (2012). A DYNC1H1 mutation causes a dominant spinal muscular atrophy with lower extremity predominance. Neurogenetics , 13, 327-332. doi: 10.1007/s10048-012-0337-6

Weedon, M. N., Hastings, R., Caswell, R., Xie, W., Paszkiewicz, K., Antoniadi, T., Williams, M., King, C., Greenhalgh, L., Newbury-Ecob, R., \& Ellard, S. (2011). Exome sequencing identifies a DYNC1H1 mutation in a large pedigree with dominant axonal Charcot-Marie-Tooth disease. Am J Hum Genet, 89, 308-312. doi: $10.1016 /$ j.ajhg.2011.07.002

\section{Figure legends}

Fig. 1 Sanger sequencing results of the two pedigrees

A, Sanger sequencing of patient 1 and her parents revealed a de novo DYNC1H1 gene mutation c.1792C>T; B, Sanger sequencing of patient 2 and his parents revealed a de novo DYNC1H1 gene mutation c.790C $>$ G; C, The pathogenicity prediction of the mutations by bioinformatics analysis based on several databases and according to ACMG guidelines.

Fig.2 Mutation spectrum of $D Y N C 1 H 1$ variants on different exons

This column illustrates the distribution of $D Y N C 1 H 1$ variants on different exons. Exon 8 is the mutational hot region with the most amount of variants, followed by exon 3 , exon 5 and exon 13, etc.

\section{Fig.3 The structural model of DYNC1H1 protein}

This figure illustrates the DYNC1H1 protein. It contains 4646 amino acids and can be roughly divided into the tail domain and the motor domain. More exactly, this protein can be divided into several domains overlapping each other, including the stem domain, DIC-binding domain, DILC-binding domain, AAA domains, the stalk domain, etc.

Abbreviations: DIC: dynein intermediate chain; DILC: dynein intermediate light chain

\section{Fig.4 The clinical features of neuromuscular diseases caused by DYNC1H1 mutations}

A, The pie chart figures the distribution of disease onset age of patients; $\mathbf{B}$, This column chart indicates the percentage of some common features of DYNC1H1- related neuromuscular diseases.

Abbreviations: LL: lower limbs; UL: upper limbs.

\section{Fig.S1 Flow chart of publication screening process}

A total of 22 previous publications out of 49 original articles were finally included according to the inclusion and exclusion criteria.

Table 1 Phenotypic comparison between SMA-LED patients and CMT2O patients

\begin{tabular}{lllll}
\hline Characteristic & SMA-LED & CMT2O & $P$ value & $P$ value \\
Female/Male & $22 / 22$ & $3 / 13$ & 0.030 & 0.030
\end{tabular}




\begin{tabular}{|c|c|c|c|c|}
\hline Mean age \pm SD (range), years & $21.86 \pm 19.88$ & $26.67 \pm 20.04$ & 0.727 & 0.727 \\
\hline Mean onset age & $30 / 63(476)$ & $2 / 15(13.3)$ & $<0,05$ & 0006 \\
\hline infantile ([?]1y) (\%) & $10 / 63(15.9)$ & $7 / 15(46.7)$ & $<0.05$ & \\
\hline early childhood (>1y, [?]3y) (\%) & $10 / 63(15.9)$ & $4 / 15(26.7)$ & $>0.05$ & \\
\hline childhood (>3y, [?]12y) (\%) & $6 / 63(9.5)$ & $1 / 15(6.7)$ & $>0.05$ & \\
\hline juvenile (>12y, [?]20y) (\%) & $0(0)$ & $1 / 15(6.7)$ & $<0.05$ & \\
\hline adulthood (>20y) (\%) & 7/63 (11.1) & $0(0)$ & $>0.05$ & \\
\hline Epilepsy (\%) & $7 / 73(9.6)$ & $0(0)$ & 0.437 & 0.437 \\
\hline Delayed motor milestones (\%) & $32 / 62(51.6)$ & $12 / 16(75.0)$ & 0.093 & 0.093 \\
\hline LL muscle atrophy (\%) & $60 / 70(85.7)$ & $13 / 16(81.3)$ & 0.950 & 0.950 \\
\hline PLL-dominant (\%) & $11 / 42(26.2)$ & $0(0)$ & $<0.05$ & 0.112 \\
\hline DLL-dominant (\%) & $24 / 42(57.1)$ & $10 / 13(76.9)$ & $>0.05$ & \\
\hline both PLL and DLL (\%) & $7 / 42(16.7)$ & $3 / 13(23.1)$ & $>0.05$ & \\
\hline mild $(\%)$ & $7 / 20(35.0)$ & $3 / 3(100)$ & $<0.05$ & 0.207 \\
\hline moderate $(\%)$ & $5 / 20(25.0)$ & $0(0)$ & $>0.05$ & \\
\hline severe $(\%)$ & $8 / 20(40.0)$ & $0(0)$ & $>0.05$ & \\
\hline LL muscle weakness (\%) & $72 / 74(97.3)$ & $16 / 16(100)$ & 1.000 & 1.000 \\
\hline PLL-dominant (\%) & $39 / 48(81.3)$ & $1 / 16(6.2)$ & $<0.05$ & $<0.001$ \\
\hline DLL-dominant (\%) & $3 / 48(6.2)$ & $13 / 16(81.3)$ & $<0.05$ & \\
\hline both PLL and DLL (\%) & $6 / 48(12.5)$ & $2 / 16(12.5)$ & $>0.05$ & \\
\hline mild (\%) & $8 / 20(40.0)$ & $5 / 6(83.3)$ & $>0.05$ & 0.239 \\
\hline moderate $(\%)$ & $8 / 20(40.0)$ & $1 / 6(16.7)$ & $>0.05$ & \\
\hline severe $(\%)$ & $4 / 20(20.0)$ & $0(0)$ & $>0.05$ & \\
\hline UL involvement (\%) & $12 / 69(17.4)$ & $3 / 16(18.8)$ & 1.000 & 1.000 \\
\hline Trunk involvement (\%) & $3 / 69(4.3)$ & $1 / 16(6.2)$ & 0.573 & 0.573 \\
\hline Abnormal tendon reflex $(\%)$ & $57 / 63(90.5)$ & $10 / 16(62.5)$ & 0.017 & 0.017 \\
\hline Decreased/absent (\%) & $55 / 57(96.5)$ & $10 / 10(100)$ & $>0.05$ & 1.000 \\
\hline Increased $(\%)$ & $2 / 57(3.5)$ & $0(0)$ & $>0.05$ & \\
\hline Ambulant (\%) & $65 / 76(85.5)$ & $15 / 16(93.8)$ & 0.632 & 0.632 \\
\hline Gower's sign (\%) & $7 / 44(15.9)$ & NA & NA & NA \\
\hline Abnormal sensation (\%) & $1 / 66(1.5)$ & $9 / 16(56.3)$ & $<0.001$ & $<0.001$ \\
\hline Deformities (\%) & $66 / 73(90.4)$ & $13 / 16(81.3)$ & 0.539 & 0.539 \\
\hline joint $(\%)$ & $32 / 66(48.5)$ & $1 / 16(6.2)$ & 0.006 & 0.006 \\
\hline feet $(\%)$ & $53 / 66(80.3)$ & $8 / 16(50.0)$ & 0.266 & 0.266 \\
\hline pes cavus $(\%)$ & $27 / 66(40.9)$ & $8 / 16(50.0)$ & 0.171 & 0.171 \\
\hline pes planus $(\%)$ & $5 / 66(7.6)$ & $0(0)$ & 0.584 & 0.584 \\
\hline others $(\%)$ & $21 / 66(31.8)$ & $2 / 16(12.5)$ & 0.391 & 0.391 \\
\hline hands (\%) & $3 / 66(4.5)$ & $0(0)$ & 1.000 & 1.000 \\
\hline spine $(\%)$ & $15 / 66(22.7)$ & $5 / 16(31.3)$ & 0.399 & 0.399 \\
\hline others $(\%)$ & $9 / 66(13.6)$ & $2 / 16(12.5)$ & 1.000 & 1.000 \\
\hline Cognitive impairment (\%) & $17 / 66(25.8)$ & $4 / 16(25.0)$ & 1.000 & 1.000 \\
\hline mild $(\%)$ & $13 / 66(19.7)$ & NA & NA & NA \\
\hline moderate $(\%)$ & $1 / 66(1.5)$ & NA & NA & NA \\
\hline severe $(\%)$ & $0(0)$ & NA & NA & NA \\
\hline $\operatorname{ADHD}(\%)$ & $9 / 66(13.6)$ & $0(0)$ & 0.263 & 0.263 \\
\hline Increased CK level (\%) & $3 / 24(12.5)$ & $\mathrm{NA}$ & NA & NA \\
\hline Abnormal NCV results (\%) & $7 / 34(20.6)$ & $0(0)$ & 0.128 & 0.128 \\
\hline Abnormal brain MRI (\%) & $8 / 18(44.4)$ & NA & NA & NA \\
\hline
\end{tabular}

\section{Abbreviations:}


ADHD: Attention deficit hyperactivity disorder; CK: Creatine kinase; CMT2O: Charcot-Marie-Tooth type 2O; DLL: Distal lower limb; LL: Lower limb; MRI: Magnetic resonance imaging; NCV: Nerve conduction velocity; PLL: Proximal lower limb; SD: Standard deviation; SMA-LED: Spinal muscular atrophy lower limb-dominant; UL: Upper limb.

Table 2 Phenotypic comparison between mutations located on the DHC_N1 domain and the DYN1 domain

\begin{tabular}{|c|c|c|c|c|}
\hline Characteristic & DHC_N1 & DYN1 & $P$ value & $P$ value \\
\hline Female/Male & $21 / 34$ & $7 / 10$ & 0.825 & 0.825 \\
\hline Mean age \pm SD (range), years & $24.90 \pm 20.28$ & $10.39 \pm 8.84$ & 0.006 & 0.006 \\
\hline Diagnosis (SMA-LED/CMT2O) & $64 / 15$ & $13 / 1$ & 0.485 & 0.485 \\
\hline Mean onset age & & & & \\
\hline at birth $(\%)$ & $26 / 67(38.8)$ & $8 / 23(34.8)$ & $>0.05$ & 0.755 \\
\hline infantile ([?]1y) (\%) & $17 / 67(25.4)$ & $10 / 23(43.5)$ & $>0.05$ & \\
\hline early childhood (>1y, [?]3y) (\%) & $11 / 67(16.4)$ & $3 / 23(13.0)$ & $>0.05$ & \\
\hline childhood (>3y, [?]12y) (\%) & $6 / 67(9.0)$ & $1 / 23(4.3)$ & $>0.05$ & \\
\hline juvenile (>12y, [?]20y) (\%) & $1 / 67(1.5)$ & $0(0)$ & $>0.05$ & \\
\hline adulthood $(>20 y)(\%)$ & $6 / 67(9.0)$ & $1 / 23(4.3)$ & $>0.05$ & \\
\hline Epilepsy (\%) & $5 / 77(6.5)$ & $6 / 24(25.0)$ & 0.030 & 0.030 \\
\hline Delayed motor milestones (\%) & $36 / 67(53.7)$ & $18 / 23(78.3)$ & 0.038 & 0.038 \\
\hline LL muscle atrophy (\%) & $64 / 77(83.1)$ & $19 / 21(90.5)$ & 0.625 & 0.625 \\
\hline PLL-dominant $(\%)$ & $11 / 46(23.9)$ & $0(0)$ & $>0.05$ & 0.032 \\
\hline DLL-dominant $(\%)$ & $25 / 46(54.3)$ & $9 / 9(100)$ & $<0.05$ & \\
\hline both PLL and DLL (\%) & $10 / 46(21.7)$ & $0(0)$ & $>0.05$ & \\
\hline mild $(\%)$ & $8 / 18(44.4)$ & $2 / 5(40.0)$ & $>0.05$ & 0.297 \\
\hline moderate $(\%)$ & $5 / 18(27.8)$ & $0(0)$ & $>0.05$ & \\
\hline severe $(\%)$ & $5 / 18(27.8)$ & $3 / 5(60.0)$ & $>0.05$ & \\
\hline LL muscle weakness (\%) & $76 / 79(96.2)$ & $22 / 23(95.7)$ & 1.000 & 1.000 \\
\hline PLL-dominant (\%) & $37 / 57(64.9)$ & $7 / 11(63.6)$ & $>0.05$ & 0.159 \\
\hline DLL-dominant (\%) & $15 / 57(26.3)$ & $1 / 11(9.1)$ & $>0.05$ & \\
\hline both PLL and DLL (\%) & $5 / 57(8.8)$ & $3 / 11(27.3)$ & $>0.05$ & \\
\hline mild (\%) & $10 / 22(45.5)$ & $4 / 12(33.3)$ & $>0.05$ & 0.048 \\
\hline moderate $(\%)$ & $8 / 22(36.4)$ & $1 / 12(8.3)$ & $>0.05$ & \\
\hline severe $(\%)$ & $4 / 22(18.2)$ & $7 / 12(58.3)$ & $<0.05$ & \\
\hline UL involvement (\%) & $13 / 73(17.8)$ & $5 / 24(20.8)$ & 0.978 & 0.978 \\
\hline Trunk involvement (\%) & $3 / 73(4.1)$ & $2 / 24(8.3)$ & 0.780 & 0.780 \\
\hline Abnormal tendon reflex (\%) & $57 / 70(81.4)$ & $18 / 20(90.0)$ & 0.571 & 0.571 \\
\hline Decreased/absent (\%) & $55 / 57(96.5)$ & $18 / 18(100)$ & $>0.05$ & 1.000 \\
\hline Increased $(\%)$ & $2 / 57(3.5)$ & $0(0)$ & $>0.05$ & \\
\hline Ambulant (\%) & $72 / 81(88.9)$ & $17 / 21(81.0)$ & 0.545 & 0.545 \\
\hline Gower's sign (\%) & $8 / 41(19.5)$ & $1 / 13(7.7)$ & 0.569 & 0.569 \\
\hline Abnormal sensation (\%) & $10 / 69(14.5)$ & $0(0)$ & 0.258 & 0.258 \\
\hline Deformities (\%) & $68 / 77(88.3)$ & $23 / 24(95.8)$ & 0.493 & 0.493 \\
\hline joint (\%) & $27 / 68(39.7)$ & $9 / 23(39.1)$ & 0.961 & 0.961 \\
\hline feet $(\%)$ & $52 / 68(76.5)$ & $15 / 23(65.2)$ & 0.290 & 0.290 \\
\hline pes cavus $(\%)$ & $32 / 68(47.1)$ & $5 / 23(21.7)$ & 0.033 & 0.033 \\
\hline pes planus (\%) & $4 / 68(5.9)$ & $2 / 23(8.7)$ & 1.000 & 1.000 \\
\hline others $(\%)$ & $18 / 68(26.5)$ & $8 / 23(34.8)$ & 0.446 & 0.446 \\
\hline hands $(\%)$ & $0(0)$ & $3 / 23(13.0)$ & 0.015 & 0.015 \\
\hline spine $(\%)$ & $16 / 68(23.5)$ & $9 / 23(39.1)$ & 0.147 & 0.147 \\
\hline others $(\%)$ & $9 / 68(13.2)$ & $11 / 23(47.8)$ & 0.001 & 0.001 \\
\hline
\end{tabular}




\begin{tabular}{lllll} 
Cognitive impairment (\%) & $16 / 75(21.3)$ & $15 / 19(78.9)$ & $<0.001$ & $<0.001$ \\
mild (\%) & $9 / 11(81.8)$ & $8 / 10(80.0)$ & $>0.05$ & 1.000 \\
moderate (\%) & $1 / 11(9.1)$ & $0(0)$ & $>0.05$ & \\
severe (\%) & $1 / 11(9.1)$ & $2 / 10(20.0)$ & $>0.05$ & \\
ADHD (\%) & $8 / 75(10.7)$ & $5 / 19(26.3)$ & 0.164 & 0.164 \\
Increased CK level (\%) & $3 / 22(13.6)$ & $0(0)$ & 1.000 & 1.000 \\
Abnormal NCV results (\%) & $4 / 45(8.9)$ & $6 / 12(50.0)$ & 0.004 & 0.004 \\
Abnormal brain MRI (\%) & $6 / 17(35.3)$ & $12 / 14(85.7)$ & 0.005 & 0.005 \\
\hline
\end{tabular}

\section{Abbreviations:}

ADHD: Attention deficit hyperactivity disorder; CK: Creatine kinase; CMT2O: Charcot-Marie-Tooth type 2O; DLL: Distal lower limb; LL: Lower limb; MRI: Magnetic resonance imaging; NCV: Nerve conduction velocity; PLL: Proximal lower limb; SD: Standard deviation; SMA-LED: Spinal muscular atrophy lower limb-dominant; UL: Upper limb.

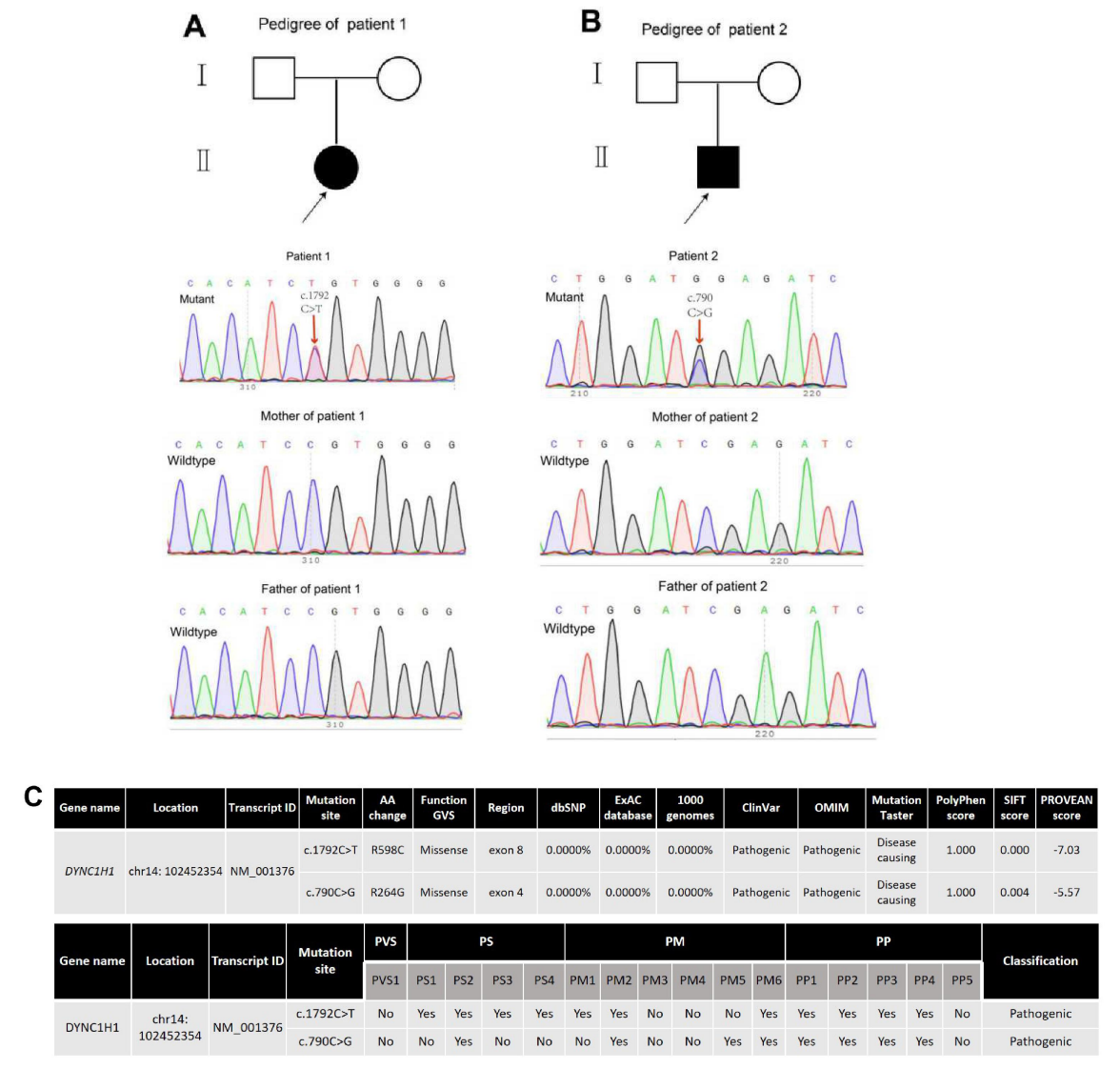

B Pedigre of patent 2 

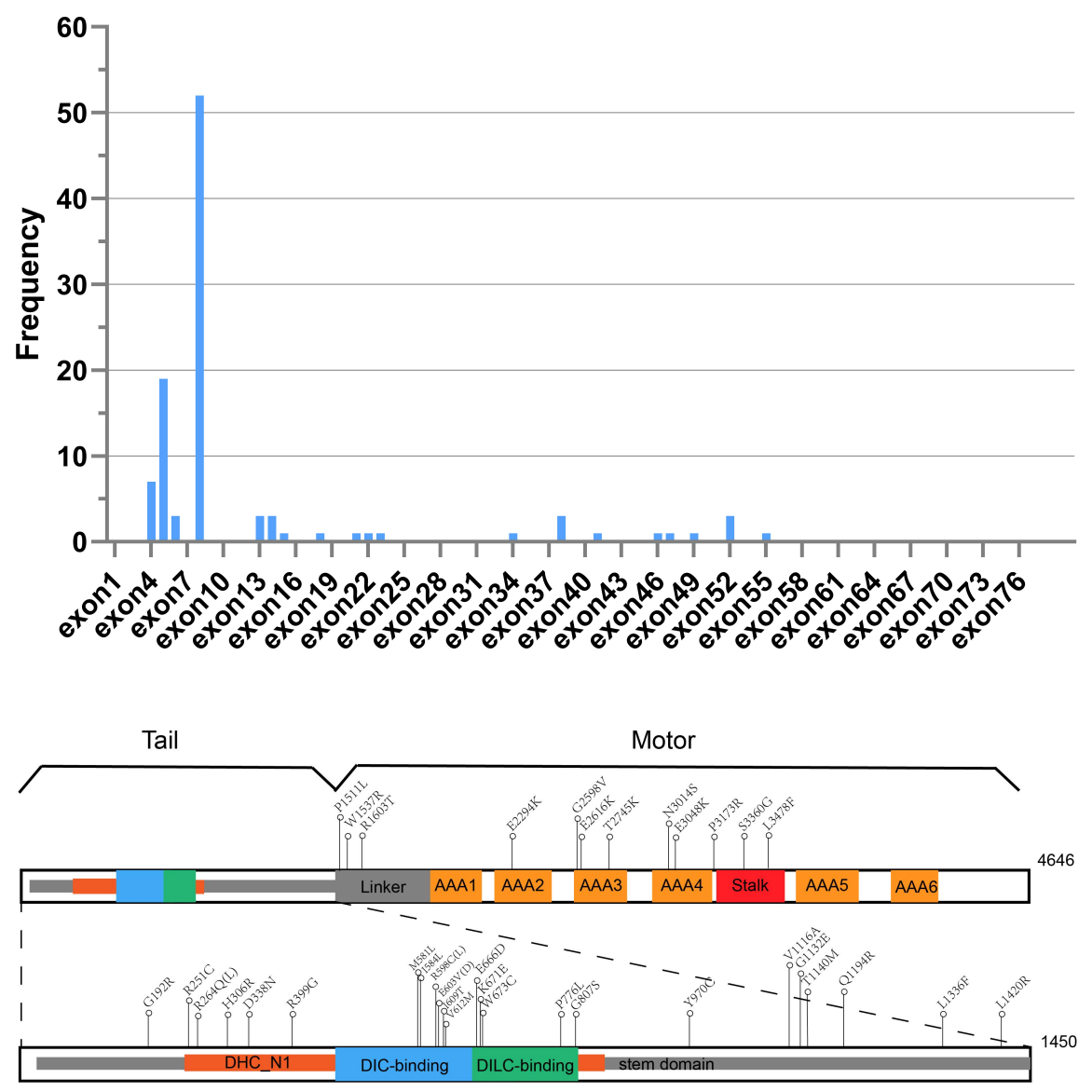

A

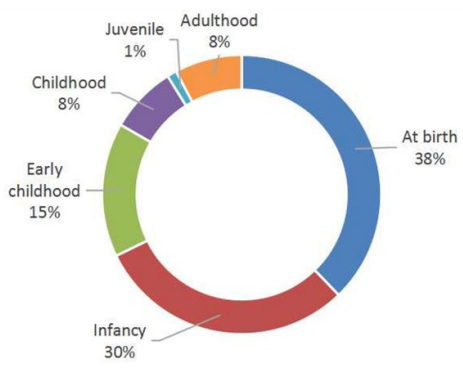

B

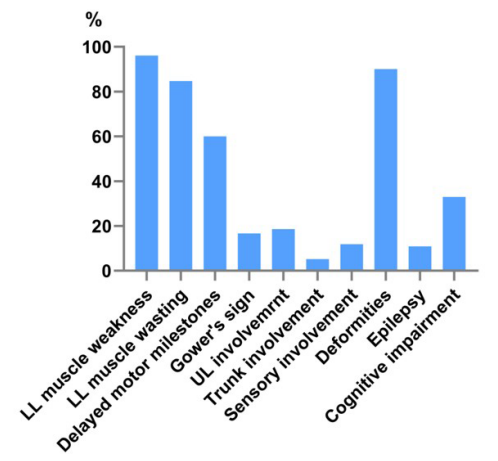

